\title{
A Catalog and Atlas of Cataclysmic Variables - The Living Edition
}

\author{
Ronald A. Downes \\ Space Telescope Science Institute, Baltimore, MD 21218 \\ downes@stsci.edu \\ Ronald F. Webbink \\ Department of Astronomy, University of Illinois, 103 Astr. Building, 1002 W. Green St., Urbana, \\ IL 61801 \\ webbink@astro.uiuc.edu \\ Michael M. Shara \\ American Museum of Natural History, Astrophysics Department, Central Park West 6 79th St., \\ New York, NY 10024 \\ mshara@amnh.org \\ Hans Ritter \\ Max-Planck-Institut für Astrophysik, Karl-Schwarzschild-Str.1, D-85741 Garching, Germany \\ hsr@mpa-garching.mpg.de \\ Ulrich Kolb \\ Department of Physics and Astronomy, The Open University, Walton Hall, Milton Keynes MK 7 \\ 6AA, United Kingdom \\ U.C.Kolb@open.ac.uk \\ and \\ Hilmar W. Duerbeck \\ WE/OBSS, Free University Brussels (VUB), Pleinlaan 2, B-1050 Brussels, Belgium \\ hduerbec@vub.ac.be
}

\begin{abstract}
The Catalog and Atlas of Cataclysmic Variables (Edition 1 - Downes and Shara (1993) and Edition 2 - Downes, Webbink, and Shara (1997)) has been a valuable source
\end{abstract}


of information for the cataclysmic variable (CV) community. However, the goal of having a central location for all objects is slowly being lost as each new edition is generated. There can also be a long time delay between new information becoming available on an object and its publication in the catalog. To eliminate these concerns, as well as to make the catalog more accessible, we have created a web site which will contain a "living" edition of the catalog. We have also added orbital period information, as well as finding charts for novae, to the catalog.

Subject headings: cataclysmic variables, catalogs, atlases

\section{Introduction}

The Catalog and Atlas of Cataclysmic Variables (Edition 1 - Downes and Shara (1993) and Edition 2 - Downes, Webbink, and Shara (1997)) has been a valuable source of information for the cataclysmic variable (CV) community. One of the goals of the catalog was to have the basic information on the objects (i.e. coordinates, type, magnitude range, and finding charts) in one central location, thus making it easy for observers to obtain data on the objects. However, the impracticality of reprinting the finding charts in their entirety means that, with each new edition, they are spread among more publications, taking us further from our goal of a central location. Furthermore, as new objects are discovered, and known ones examined in greater detail, the printed editions cannot keep pace with discovery, A "living" edition is therefore highly desirable, so that observers can access a complete and current list of CVs at any time.

For the above reasons, as well as the need to simplify the tracking of the objects (there are over 1200 objects in the catalog), we have decided to generate a web-based version of the catalog. This version will have all the information (as well some additional information detailed below) from the first two editions, plus information on over 150 new objects discovered since 1996 May. Those objects with revised finding charts will only have one chart presented, thus eliminating a possible confusion which necessarily exists when "paper" catalogs are generated. The web site will also allow for easy searching of the catalog, and for generation of basic statistics (e.g. how many dwarf novae, how many CVs have Hubble Space Telescope data, etc.).

The catalog consists of (as of 2000 December) 1034 CVs, and another 194 objects that are noncvs (objects originally classified erroneously as CVs). Most of the objects are dwarf novae (40\%), with another $30 \%$ being novae, and the rest mostly novalike variables. A large fraction (90\%) of the CVs have references to published finding charts, while $64 \%$ of the objects have published spectra (49\% quiescent spectra and $15 \%$ outburst spectra).

We have taken this opportunity to make several enhancements to the catalog. In conjunction with Hans Ritter and Ulrich Kolb, we have added orbital period data to the catalog; about one-third

of the objects have periods. The period information is from Ritter and Kolb (1998), plus updated 
and additional values. In conjunction with Hilmar Duerbeck (Duerbeck 1987), we now include finding charts of novae (when possible), and have measured coordinates for many in the Hubble Space Telescope GSC v1.1 Guide Star Reference frame (as is the case for the non-novae). Finally, in the first edition we introduced (out of necessity) a pseudo-GCVS name for certain objects (e.g. Phe1), which was continued in the second edition. With the web-based catalog, these names are no longer needed, so we will cease generating new ones. For those objects that already had such names (some of which have appeared in subsequent papers in the literature) and now have a formal GCVS designation, we will adopt the formal GCVS name, although we will keep the pseudo-GCVS name in the "Other Name" field for continuity.

\section{The Site}

The site can be reached via:

http://icarus.stsci.edu/ downes/cvcat/

and is described in detail below.

\subsection{Home Page}

The Home Page (Figure 1) for the catalog contains six links:

- Search - a link to the Search Page, from which the catalog may be accessed.

- Description - a description of the catalog, following the format of the previous editions. A description of all the fields is given.

- References - a complete listing of the references mentioned in the catalog. Note that from each Individual Object Page, you can go directly to the reference of interest.

- Statistics - a listing of a fixed set of basic statistics from the catalog, generated in real-time.

- ASCII report - a listing of the entire catalog in the format of the previously published versions (i.e. containing most but not all of the fields), sorted by right ascension. This output can be down-loaded to an ASCII file.

- Change log - a listing, by object, of the changes made since the initial release of this edition

\subsection{Search Page}

The Search Page (Figure 2) is the main page for access to the catalog. It allows the user to search the catalog on any field or combination of fields. The following text fields can be searched in a 
case-insensitive manner: GCVS Name, Other Name, and the five reference fields (coordinate, chart, type, spectrum, and period); the Object Type and Notes fields can be searched in a case-sensitive manner. All textual searches support the use of wildcards. A coordinate search may be performed by specifying either a right ascension/declination range, or by specifying a set of coordinates and a radius. Numerical searches (supporting a " $<$ " and " $>$ " capability) can be performed for the following fields: galactic latitude, minimum and maximum magnitude, outburst year (for novae), and period. Finally, a search for space-based observations using any of 10 observatories can be

performed. An on-line help file is available detailing the search capabilities for each field, as well as providing instructions for the use of wildcards.

\subsection{Search Results Page}

After a search is initiated, the Search Results Page (Figure 3) presents the results of the search. This page indicates the number of objects in the catalog that match the selection criteria, and presents an abbreviated view of the catalog entries for such entries, showing the basic information such as the coordinates, type, magnitude range, and period. To obtain the full information (including the finding chart), one clicks on the object of interest.

\subsection{Individual Object Page}

The Individual Object Page (Figure 4) presents the complete information on the selected object. For the finding charts, the field size, source (DSS (Digitized Sky Survey), Hubble Space Telescope data, ground-based image), filter/emulsion, and exposure time are given to allow the user to estimate the depth of the image. For most objects, the DSS image is used. However, for particularly crowded fields (such as in globular clusters), Hubble Space Telescope data is used when available. Similarly, for particularly faint targets, ground-based CCD images are provided when possible. On this page, one may click on any of the reference codes to go directly to the full reference on the References page.

\section{Site Maintainance}

We plan to update the site with new objects and information on a continual basis, although period and spaced-based updates will occur roughly every six months. We encourage users to inform us of any updates that should be implemented (e.g. revised identifications, new objects, etc.), and if appropriate to send us improved/original finding charts (as either postscript or jpeg images). The charts will be particularly useful for recent novae recovered in quiescence, and for the faintest objects where deep CCD imaging clearly reveals the correct identification. 
We wish to thank Anne Gonnella, Steve Hulbert, Calvin Tullos, and Mike Wiggs for the excellent work in creating the site. We also wish to thank Matt McMaster for assistance in generating the multitude of finding charts, and the Director's Discretionary Research Fund at STScI for financial support. Paula Szkody, John Thorstensen, and Steve Howell provided helpful comments on the initial version of the site. RFW gratefully acknowledges the support of NSF grant AST-9618462, and sabbatical support from STScI. HWD acknowledges the hospitality and support of STScI.

\section{REFERENCES}

Downes, R.A. and Shara, M.M. 1993, PASP, 105, 127

Downes, R.A., Webbink, R.F., and Shara, M.M. 1997, PASP, 109, 345

Duerbeck, H.W. 1987, Ap\&SS, 131, 461

Ritter, H. and Kolb, U. 1998, A\&AS, 129, 83 
A Catalog and Atlas of Cataclysmic Variables Living Edition

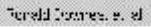

Scarch | Deseription| Reforences

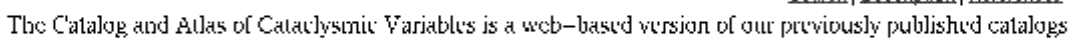
(Dowiocs and Shard 1993, PASP 105, 127; Down's, Wobbiok and Shard 1997. PASP 100, 345). The dala

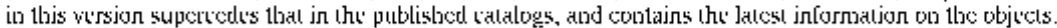

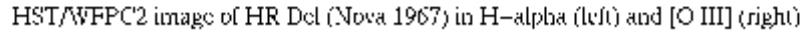
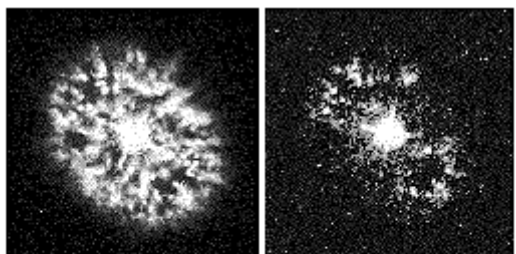

Scarch Look for trutrics is the tatalog using any of the cala]og fiteds as st'dt'h crilcria. Results include a PDF virsion [or casjer printiog.

Description Read about the cala]og, the data it contains, and destriptions of the fiteds.

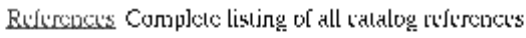

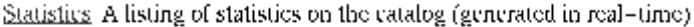

BSCII repoct A listing of the tatalog is the format of the publisbed sersions, sotted by RA. which can be dowis-loaded to als ASCII fils

Chang: Lop A listing, by object, of the thanges made sinte the initjal twease of the cililoles.

Fig. 1.- The site Home page.

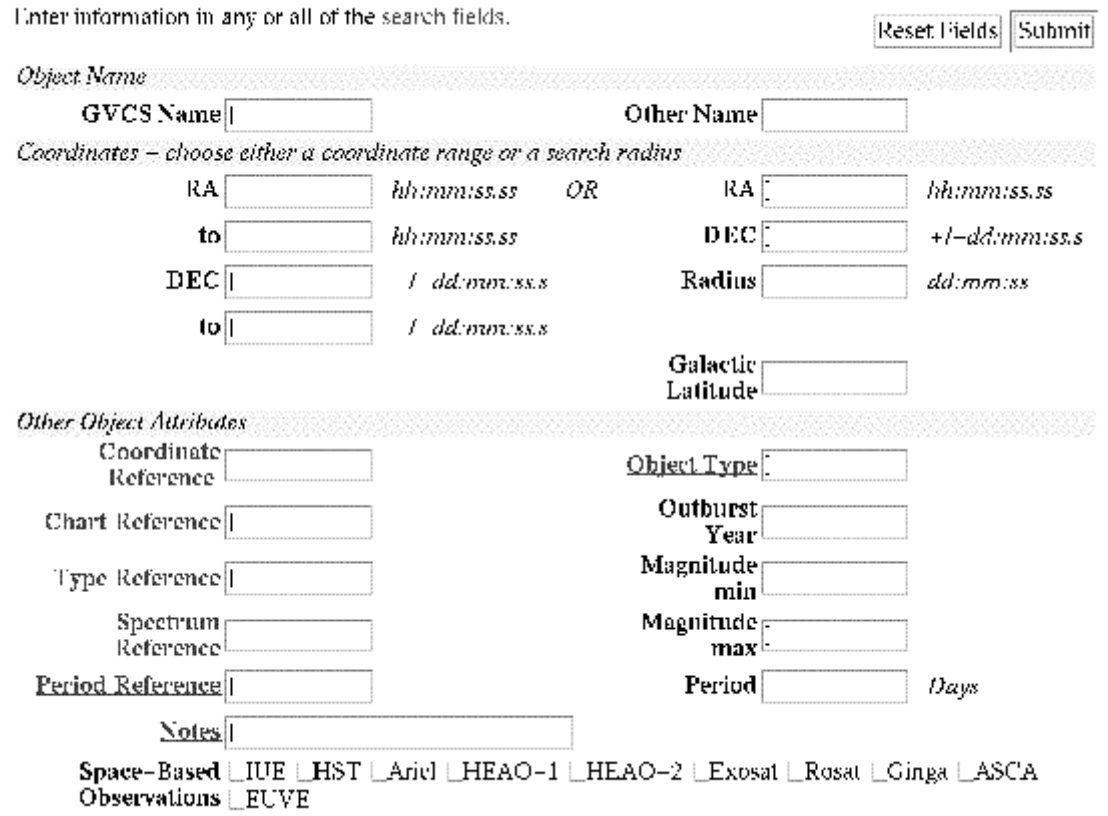

Fig. 2. - The Search page. 


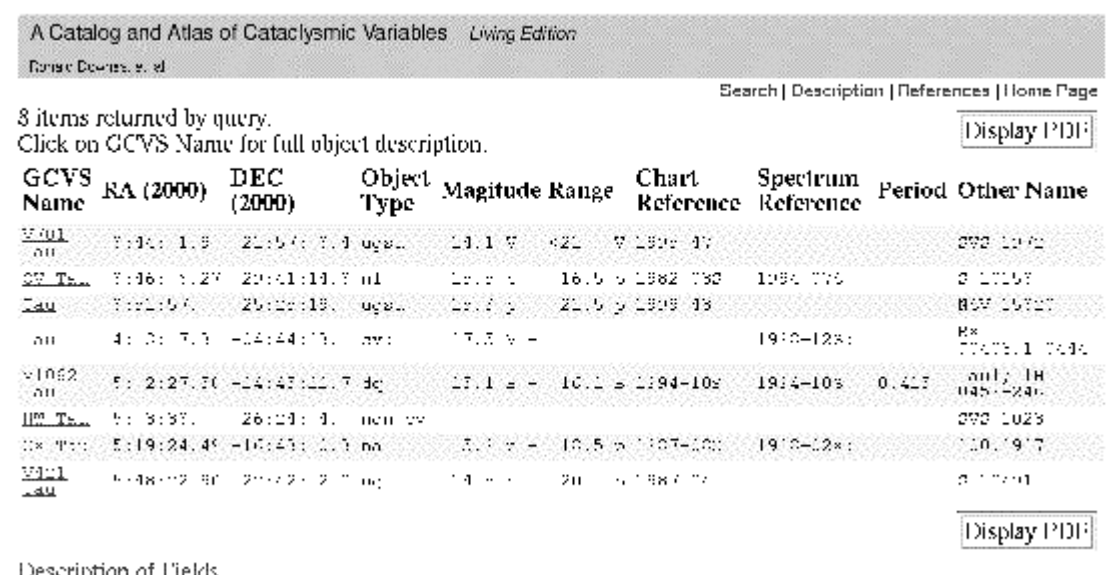

Fig. 3. - The Search Results page.

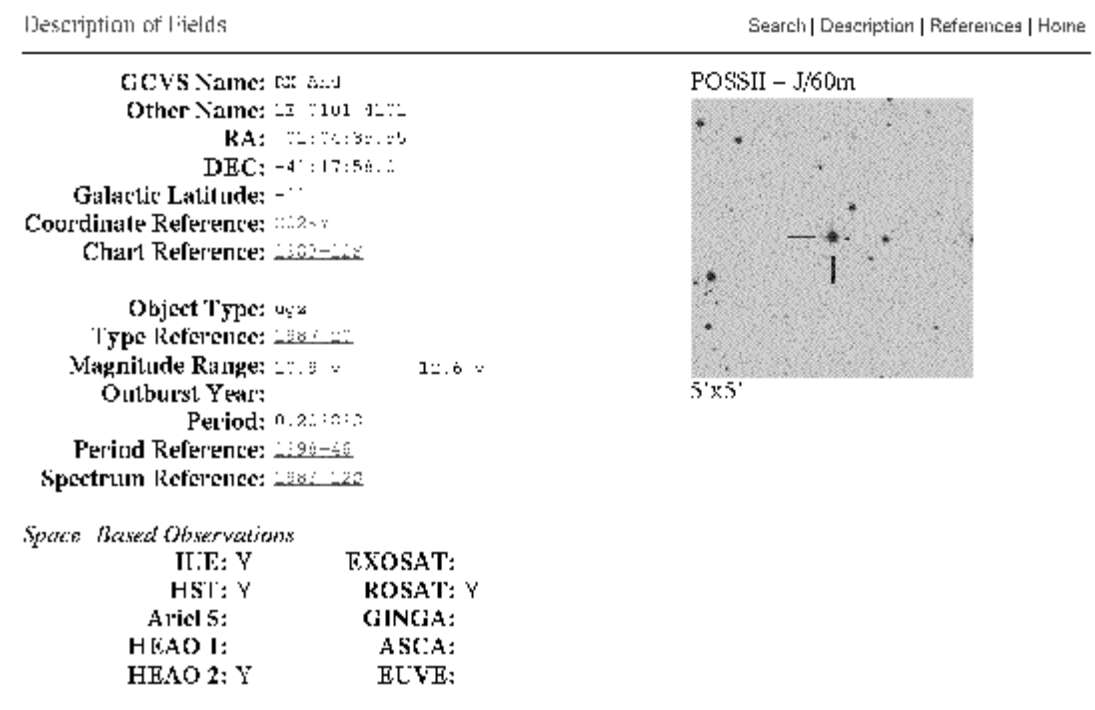

Notes: IISI data is spectroscopic (GIIRS)

Lasi Lpdated: F'b 122001

Jescription of liields

Fig. 4.- The Individual Object page. 